\title{
Comparison of the Effects of Acute and Chronic Administration of Tetrahydroisoquinoline Amines on the In Vivo Dopamine Release: A Microdialysis Study in the Rat Striatum
}

\author{
Agnieszka Wąsik ${ }^{1} \cdot$ Irena Romańska $^{1}$ Lucyna Antkiewicz-Michaluk ${ }^{1}$
}

Received: 14 June 2016/Revised: 11 August 2016/Accepted: 13 August 2016/Published online: 27 August 2016

(C) The Author(s) 2016. This article is published with open access at Springerlink.com

\begin{abstract}
The etiology of Parkinson's disease (PD) may involve endogenous and exogenous factors. 1-Benzyl-1,2,3,4tetrahydroisoquinoline (1BnTIQ), which was shown to be neurotoxic for dopaminergic neurons, is one of such factors, thus it can be used to construct an animal model of PD. In contrast, 1,2,3,4-tetrahydroisoquinoline (TIQ) and 1-methyl1,2,3,4-tetrahydroisoquinoline (1MeTIQ) produce neuroprotective effects acting as monoamino oxidase (MAO) inhibitors and free radical scavengers that reduce oxidative stress in the mammalian brain. In this study, we aimed to investigate the effects of neuroprotective compounds, TIQ and 1MeTIQ, on the dopamine release in vivo in an animal model of PD induced by chronic administration of 1BnTIQ $(25 \mathrm{mg} / \mathrm{kg}$ i.p.). Using an in vivo microdialysis methodology, we measured the impact of both acute and chronic treatment with TIQ and 1MeTIQ (50 mg/kg i.p.) on 1BnTIQ-induced changes in dopamine release in the rat striatum. Additionally, the behavioral test was carried out to check the influence of repeated administrations of the investigated compounds on the locomotor activity of rats. The behavioral studies showed that the chronic administration of 1BnTIQ produced a significant elevation of exploratory locomotor activity, and both the investigated amines, TIQ and 1MeTIQ, administered together with 1BnTIQ completely prevented 1BnTIQ-produced hyperactivity. The in vivo microdialysis studies demonstrated that the chronic treatment with 1BnTIQ caused a significant and long-lasting increase in the dopamine release (approximately $300 \%$ ) to the extracellular space in the rat
\end{abstract}

Agnieszka Wąsik

wasik@if-pan.krakow.pl

1 Department of Neurochemistry, Institute of Pharmacology, Polish Academy of Sciences, Smętna 12, 31-343 Kraków, Poland striatum, which was demonstrated in the basal samples $24 \mathrm{~h}$ after 1BnTIQ injection. The combined chronic administration of 1BnTIQ and the investigated compounds, TIQ or 1MeTIQ, completely antagonized the 1BnTIQ-induced essential disturbances of the dopamine releasing to the extracellular space in the striatum. In conclusion, we suggest that higher concentrations of 1BnTIQ in the brain produced distinct impairment in the dopamine release, whereas TIQ and 1MeTIQ (compounds with previously revealed neuroprotective properties) completely prevented 1BnTIQ-induced abnormalities in the function of dopamine neurons and restored the dopamine release to the control values.

Keywords Tetrahydroisoquinolines - Dopamine release . Microdialysis study $\cdot$ Rat striatum

\section{Introduction}

Tetrahydroisoquinolines are a group of endogenous compounds that are present in the mammalian brain (Abe et al. 2005; Yamakawa et al. 1999; Yamakawa and Ohta 1997, 1999). Some of these compounds, such as 1-benzyl1,2,3,4-tetrahydroisoquinoline (1BnTIQ), have shown neurotoxic properties and are considered to be involved in the pathogenesis of Parkinson's disease (PD) (Abe et al. 2001a; Kotake et al. 1995). Kotake et al. (1995) indicated that the concentration of 1BnTIQ in the cerebrospinal fluid (CSF) of parkinsonian patients was three times higher than that in the CSF of the control group. Previous studies revealed that chronic treatment with 1BnTIQ induces parkinsonian-like symptoms in mammals (Kotake et al. 1995, 2003, 2010). 1BnTIQ accumulates in dopaminergic neurons and can lead to parkinsonian symptoms. 1BnTIQ evoked strong activation of the oxidative MAO-dependent 
catabolic pathway (Wạsik et al. 2009). In addition, 1BnTIQ significantly inhibits the COMT-dependent O-methylation pathway. This mechanism of action leads to an increase in dopamine oxidation, and as a consequence, leads to an increase in reactive oxygen species (ROS) formation in dopaminergic neurons. Moreover, in vitro studies revealed that $1 \mathrm{BnTIQ}$ induces cell death via apoptosis and produces an increase in the formation of the active caspase- 3 protein fragments (Shavali and Ebadi 2003). These data suggest that multiple administrations of 1BnTIQ might serve as an adequate animal model of the progressive process of PD. In contrast, 1,2,3,4-tetrahydroisoquinoline (TIQ), and especially its methyl derivative, 1-methyl-1,2,3,4-tetrahydroisoquinoline (1MeTIQ), have shown neuroprotective effects in the brain (Abe et al. 2001b; Antkiewicz-Michaluk et al. 2003, 2004, 2006; Wąsik et al. 2016). TIQ and $1 \mathrm{MeTIQ}$ are reversible MAO inhibitors that strongly block the MAO-dependent oxidative pathway and simultaneously increase the COMT-dependent O-methylation catabolic pathway. Therefore, both substances possess antioxidant properties. These compounds inhibit free radical formation and abolish $\mathrm{H}_{2} \mathrm{O}_{2}$ generation from dopamine via the Fenton reaction (Singer and Ramsay 1995; Antkiewicz-Michaluk et al. 2006; Patsenka and Antkiewicz-Michaluk 2004). Additionally, 1MeTIQ acts as a natural scavenger of free radicals. From a clinical point of view, the lack of a tolerance for its neuroprotective action after chronic treatment is both interesting and important (Antkiewicz-Michaluk et al. 2001; Wąsik et al. 2016).

To continue our previous studies, we would like to investigate the effects of TIQ and 1MeTIQ, as earlier demonstrated neuroprotective compounds, on the dopamine release in vivo in an animal model of PD induced by chronic administration of $1 \mathrm{BnTIQ}$. Using in vivo microdialysis methodology, we measured the impact of acute and chronic treatment with TIQ and 1MeTIQ on 1BnTIQ-induced disorders of dopamine release in the rat striatum. In addition to the biochemical research, the behavioral test was carried out to check the influence of repeated administration of 1BnTIQ on the motor activity of rats.

\section{Materials and Methods}

\section{Animals and Treatments}

All experiments were carried out in male Wistar rats with an initial body weight of 280-300 g. All animals had free access to the standard laboratory food and tap water and were kept at room temperature $\left(22^{\circ} \mathrm{C}\right)$ under an artificial light/dark cycle $(12 / 12 \mathrm{~h}$, light on at 7:00). The rats used for the microdialysis study after surgery were housed for several days individually. The experiments were carried out between 09:00 and 16:00 h. Control rats were treated with an appropriate solvent $(0.9 \% \mathrm{NaCl})$.

1BnTIQ was administered at a dose of $25 \mathrm{mg} / \mathrm{kg}$ intraperitoneally (i.p.) and chronically for 14 consecutive days. In the mixed group, TIQ or $1 \mathrm{MeTIQ}$ (50 mg/kg i.p.) was administered once $20 \mathrm{~min}$ before the last $1 \mathrm{BnTIQ}$ administration or was administered chronically $20 \mathrm{~min}$ before each 1BnTIQ injection.

All experimental procedures were performed in accordance with the National Institutes of Health Guide for the Care and Use of Laboratory Animals and were approved by the Bioethics Commission as compliant with the Polish Law. All experimental procedures were approved by the Local Bioethics Commission of the Institute of Pharmacology, Polish Academy of Sciences in Kraków.

\section{Drugs}

1BnTIQ and 1MeTIQ were synthesized (according to Cannon and Webster 1958) at the Department of Drug Chemistry of the Institute of Pharmacology, the Polish Academy of Sciences in Krakow. The purity of the compounds was verified by measuring the melting point, and the homogeneity was assessed on a chromatographic column. TIQ (Sigma-Aldrich, USA) was obtained commercially. The compounds were dissolved in a $0.9 \% \mathrm{NaCl}$ solution.

\section{Behavioral Study}

\section{Locomotor Activity}

Locomotor activity was assessed in actometers (Opto-Varimex activity monitors; Columbus Inst., USA) linked on-line to a compatible IBM PC. Each cage $(43 \times 44 \times 25 \mathrm{~cm})$ perimeter was lined with an array of $15 \times 15$ photocell beams located $3 \mathrm{~cm}$ from the floor. Interruptions of the photocell beams were counted as a measure of horizontal locomotor activity which was defined as the distance traveled (in $\mathrm{cm}$ ). Rats were given $25 \mathrm{mg} / \mathrm{kg} 1$ BnTIQ i.p. as their last dose (14-day chronic treatment group). In the combined groups, TIQ or 1MeTIQ (50 mg/kg i.p.) was given chronically $20 \mathrm{~min}$ before each 1BnTIQ administration. Subsequently, the animals were transferred to experimental cages, and locomotor activity (horizontal activity, traveled distance in $\mathrm{cm}$ ) was recorded for $90 \mathrm{~min}$ and analyzed using the AutoTrack Software Program (Columbus Instruments, USA). Each group comprised seven animals.

\section{In Vivo Microdialysis}

Rats were anesthetized with ketamine $(75 \mathrm{mg} / \mathrm{kg})$ and xylazine $(10 \mathrm{mg} / \mathrm{kg})$ and secured in a stereotaxic frame (Stoelting, USA). Vertical microdialysis guide cannulas 
(Intracerebral Guide Cannula with stylet; BAS Bioanalytical, USA) were implanted in the striatum (STR) according to the following stereotaxic coordinates: $\mathrm{A} / \mathrm{P}+1.0, \mathrm{~L} / \mathrm{M}$ +2.5 , and V/D $-3.5 \mathrm{~mm}$ from bregma and the dura $(\mathrm{G}$. Paxinos and C.H. Watson). Fourteen days after surgery, microdialysis probes were inserted into the cannulas, and the striatum was perfused with an artificial cerebrospinal fluid (aCSF), which consisted of $140 \mathrm{mM} \mathrm{NaCl}, 2.7 \mathrm{mM}$ $\mathrm{KCl}, 1.2 \mathrm{mM} \mathrm{CaCl}_{2}, 1 \mathrm{mM} \mathrm{MgCl}, 0.3 \mathrm{mM} \mathrm{NaH}_{2} \mathrm{PO}_{4}$, and $1.7 \mathrm{mM} \mathrm{Na}_{2} \mathrm{HPO}_{4}(\mathrm{pH} \mathrm{7.4})$, at a flow rate of $1.5 \mu \mathrm{l} / \mathrm{min}$ with a microinfusion pump (Stoelting, Illinois USA). Samples were collected from freely moving rats in 20-min intervals after a 3-h wash-out period. 1BnTIQ was injected chronically (for 14 consecutive days) in a $25 \mathrm{mg} / \mathrm{kg}$ i.p., and dialysis samples were collected for $180 \mathrm{~min}$. In the mixed groups, 1MeTIQ or TIQ (50 mg/kg i.p.) was administered $20 \mathrm{~min}$ before the last dose of $1 \mathrm{BnTIQ}$ (acute) or $20 \mathrm{~min}$ before each 1BnTIQ injection (chronic). All dialysates were immediately frozen on the dry ice $(-70$ ${ }^{\circ} \mathrm{C}$ ), until they were used in a biochemical assay.

Levels of dopamine (DA) and its extraneuronal metabolite, 3-methoxytyramine (3-MT), were assayed in dialysates $(20 \mu \mathrm{l})$ using HPLC with electrochemical detection as described below. The chromatograph Dionex Ultimate 3000 (Coulochem III, Germany) was equipped with C18 columns (Hypersil Gold; $150 \mathrm{~mm} \times 3 \mu$ ). The mobile phase comprised $0.05 \mathrm{M}$ citrate-phosphate buffer (pH 3.5), 0.1 mM EDTA, $1 \mathrm{mM}$ sodium octyl sulfonate, and $3.5 \%$ methanol. The flow rate was maintained at $0.75 \mathrm{ml} / \mathrm{min}$. Chromatographic data were processed using the Chromeleon Dionex computer programme (Germany). Dopamine and its metabolites were quantified by chromatograph peak height and compared with standards run on the day of the analysis. At the end of the experiment, frozen brains were examined histologically for correct probe placement. Each group consisted of six animals.

\section{Calculations and Statistics}

Data obtained from the locomotor activity and microdialysis studies were analyzed by a two-way analysis of variance (ANOVA) for repeated measures, followed by Duncan's post hoc test, if significant differences were detected.

\section{Results}

\section{The Effect of Chronic Administration of TIQ on 1BnTIQ-Induced Hyperactivity in Rats}

The multiple 1 BnTIQ (25 mg/kg i.p.) administrations produced a significant increase in the horizontal locomotor activity of rats $(\mathrm{F}[1,24]=16.30 ; p<0.01)$, while chronic administration of TIQ (50 mg/kg i.p.) induced a significant reduction in the locomotor activity of rats $(\mathrm{F}[1,24]=$ 18.29; $p<0.01)$. In the combined group, TIQ completely antagonized 1BnTIQ-induced hyperactivity (Fig. 1a).

\section{The Effect of Chronic 1MeTIQ Administration on 1BnTIQ-Induced Hyperactivity in Rats}

As shown in Fig. 1b, the multiple (14-day) administrations of $1 \mathrm{BnTIQ}$ at a low dose of $25 \mathrm{mg} / \mathrm{kg}$ (i.p.) significantly increased $(\mathrm{F}[1,24]=9.17 ; p<0.01)$ the locomotor activity, while the chronic administration of 1MeTIQ $(50 \mathrm{mg} / \mathrm{kg}$ i.p.) over 14 days induced a significant $(\mathrm{F}[1,24]=10.65$; $p<0.01)$ decrease in this parameter. In the joint $1 \mathrm{BnTIQ}+1 \mathrm{MeTIQ}$ treatment group, rats showed the response similar to that for TIQ, i.e., 1MeTIQ completely antagonized the 1BnTIQ-induced hyperactivity (Fig. 1b).

\section{The Effect of Chronic 1BnTIQ Administration on the dopamine release in vivo}

The mean control basal extracellular concentration of dopamine in dialysates obtained from the striatum was approximately $17.8 \pm 2.3(\mathrm{pg} / 20 \mu \mathrm{l})$. A statistical analysis demonstrated a significant increase (aprox. $300 \%$; $\mathrm{F}[1,17]=16.54 ; p<0.01)$ in dopamine concentration in the synaptic cleft in the basal samples (from -60 to $0 \mathrm{~min}$.) $24 \mathrm{~h}$ after chronic 1BnTIQ (25 mg/kg i.p.) administration. The last dose of 1BnTIQ fortified this effect approximately $200 \%$, and after $60 \mathrm{~min}$, the concentration of dopamine returned to the value which was observed in the basal samples but it still was elevated about $300 \%$ in comparison with the control group (saline) (Figs. 2a, b, 4a, b).

\section{The Effect of a Single or Chronic Administration of TIQ on 1BnTIQ-Induced Changes on the Dopamine Release in the Rat Striatum}

After acute TIQ administration, an increase in the dopamine release was observed (approximately $300 \%$ ) (Fig. 2a). A post hoc analysis demonstrated that chronic 1BnTIQ administration, co-administered with acute TIQ, showed a significant increase in dopamine release in the striatum of approximately $300 \% \quad(\mathrm{~F}[1,16]=18.18$; $p<0.01$ ) of the basal samples (Fig. 2a). However, after the last dose of 1BnTIQ and TIQ, the dopamine release increased up to $400 \%$ within a short period (Fig. 2a).

In contrast to the effect of chronic 1BnTIQ, chronic administration of TIQ did not change the extracellular dopamine concentration estimated $24 \mathrm{~h}$ after the last injection (the basal samples; $F[1,16]=0.47$; N.S.), but it increased the dopamine release after the last dose of TIQ 
Fig. 1 The effect of chronic administration of TIQ (a) or 1MeTIQ (b) on 1BnTIQinduced hyperactivity in rats. Rats received a single injection of saline (control). 1BnTIQ was administered at a dose of $25 \mathrm{mg} / \mathrm{kg}$ i.p. chronically for 14 consecutive days. In the mixed group, TIQ (a) or 1MeTIQ (b) $(50 \mathrm{mg} / \mathrm{kg}$ i.p. $)$ was chronically administered 20 min before each 1BnTIQ injection. Rats were placed into actometers immediately after the treatment. Movements were recorded for $45 \mathrm{~min}$. The data are expressed as the mean $\pm \operatorname{SEM}$ ( $n=6$ animals). The data were analyzed with a two-way repeated measures ANOVA, followed by Duncan's post hoc test. Statistical significance: $* p<0.05$, $*^{*} p<0.01$ versus saline-treated group; ${ }^{+} p<0.05,{ }^{++} p<0.01$ versus $1 \mathrm{BnTIQ}$-treated group
A

Locomotor activity

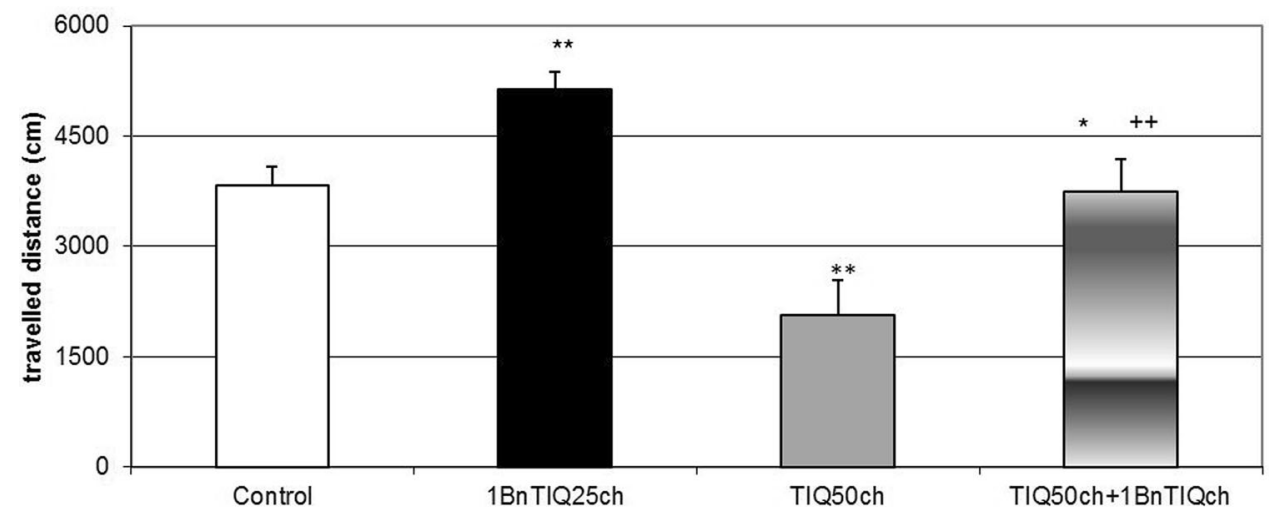

B

Locomotor activity

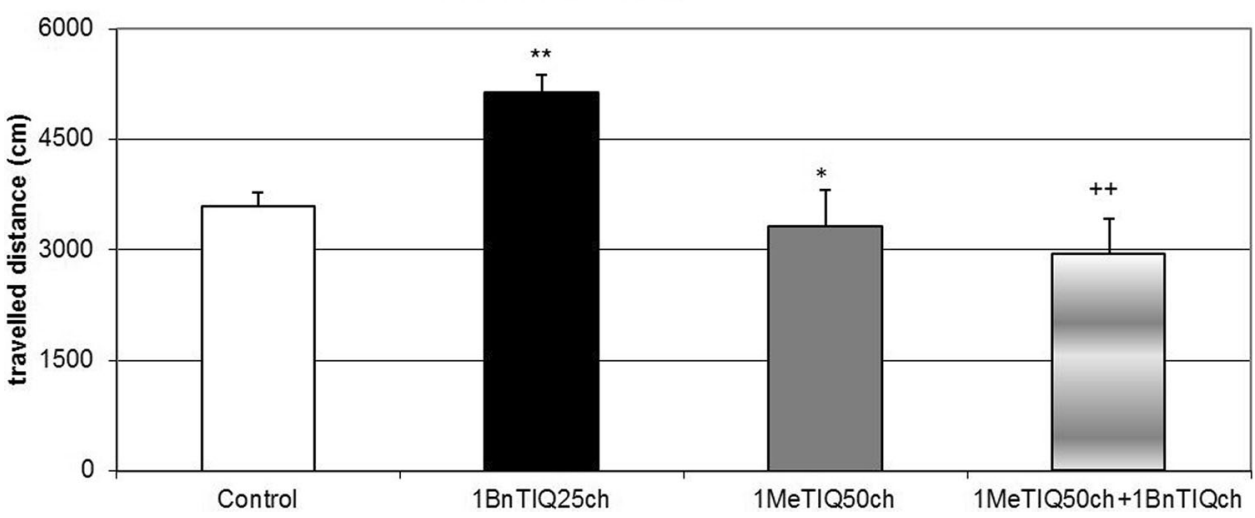

(up to $500 \%$ ) (Fig. 2b). Interestingly, in the combined treatment group, chronic administration of TIQ completely blocked the effect induced by multiple administrations of 1BnTIQ (Fig. 2b). The dopamine concentration in the basal samples was similar to that in the control (saline group); however, after the last injection of TIQ and 1BnTIQ, the dopamine release was increased by approximately $300 \%(\mathrm{~F}[1,16]=15.38$; $p<0.01$ ) (Figs. 2b).

\section{The Effect of a Single or Chronic Administration of TIQ on 1BnTIQ-Induced Changes on the Concentration of 3-MT in the Rat Striatum}

As shown in Fig. 3a, acute TIQ (50 mg/kg i.p.) administration produced a significant $(\mathrm{F}[1,16]=19.14 ; p<0.05)$ increase in the concentration of 3-MT in the rat striatum (up to $450 \%$ ). This effect was strongly potentiated in the combined treatment group (chronic 1BnTIQ + acute TIQ), (the concentration of 3-MT was elevated approximately $2500 \% ; \mathrm{F}[1,16]=11.84 ; p<0.01)($ Fig. 3a).
The post hoc analysis indicated that chronic TIQ (50 mg/kg i.p.) administration produced a huge and significant $(\mathrm{F}[1,16]=37.61 ; p<0.01)$ increase in the concentration of 3-MT in the rat striatum (up to $3500 \%$ ) (Fig. 3b). In the combined treatment group (with chronic treatment TIQ and 1BnTIQ), this effect was weaker, and the concentration of 3-MT was elevated up to $2500 \%$ $(\mathrm{F}[1,16]=30.94 ; p<0.01)($ Fig. 3b).

\section{The Effect of a Single or Chronic Administration of $1 \mathrm{MeTIQ}$ on 1BnTIQ-induced Changes in the Dopamine Release in the Rat Striatum}

An acute dose of $1 \mathrm{MeTIQ}$ produced an increase in the release of dopamine up to $200 \%$ (Fig. 4a). Duncan's test indicated that chronic $1 \mathrm{BnTIQ}+$ acute $1 \mathrm{MeTIQ}$ treatment induced a significant increase in the dopamine release in the rat striatum approximately $300 \%(\mathrm{~F}[1,17]=10.94$; $p<0.01$ ) of the basal samples (Fig. 4a). However, after the last dose of 1BnTIQ combined with an acute dose of $1 \mathrm{MeTIQ}$, we observed a reduction in the release of dopamine to $200 \%$ of the basal samples (Fig. 4a). 
Fig. 2 The effect of acute (a) or chronic (b) administration of TIQ on 1BnTIQ-induced changes on dopamine release in the rat striatum, 1BnTIQ was administered chronically (1BnTIQch) at a dose of $25 \mathrm{mg} /$ $\mathrm{kg}$ i.p. for 14 consecutive days. In the mixed group, TIQ $(50 \mathrm{mg} / \mathrm{kg}$ i.p.) was given acutely (TIQa) a 20 min before the last 1BnTIQ administration or chronically (TIQch), b 20 min before each 1 BnTIQ administration. The control group was treated with saline. The dialysate was collected every $20 \mathrm{~min}$. The concentration of dopamine (DA) was measured. The data are expressed as the mean \pm SEM $(n=5-6)$.

Statistical significance: $* p<0.05, * * p<0.01$ from the basal value; ${ }^{+} p<0.05$ versus 1BnTIQ-treated group (Duncan's test)

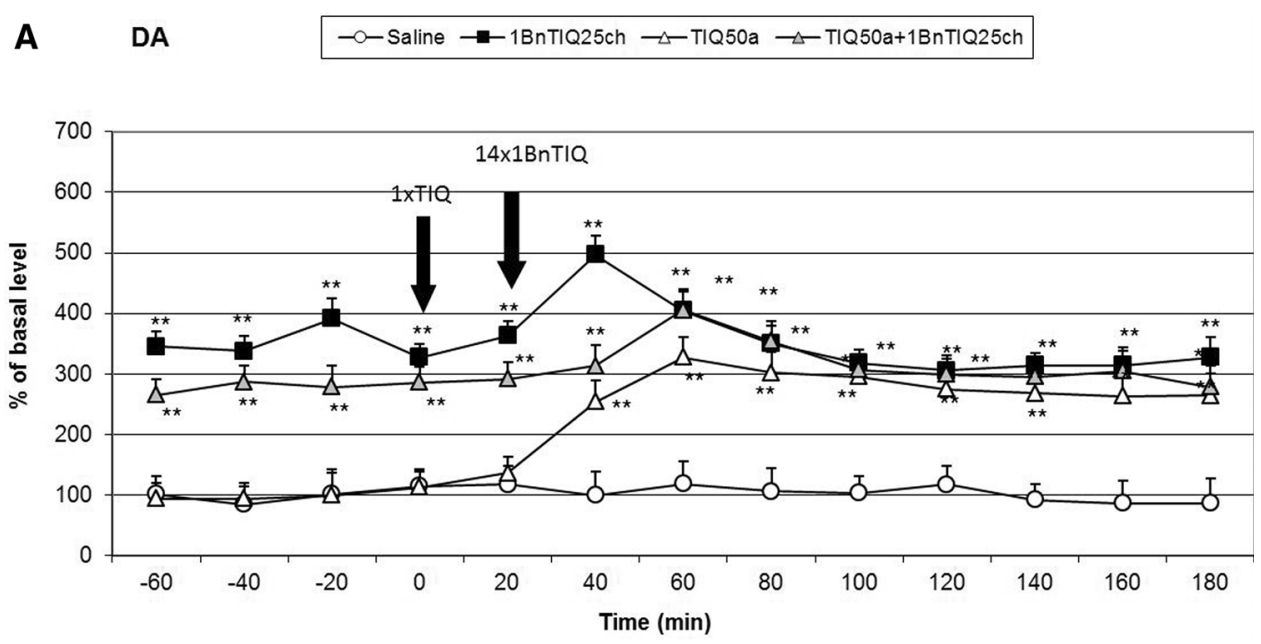

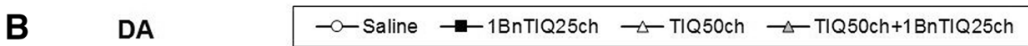

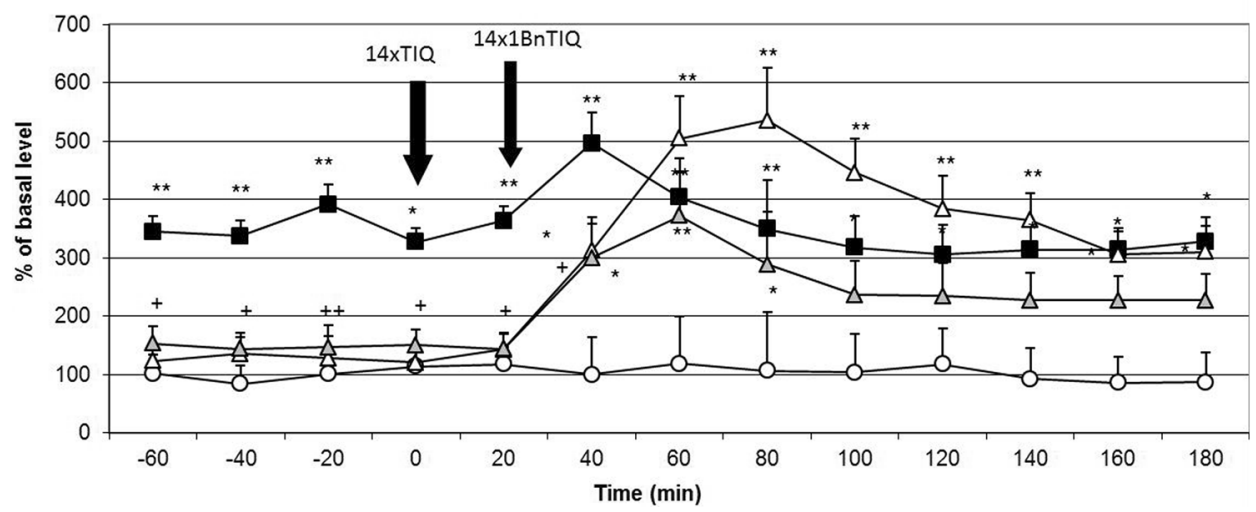

Chronic treatment with 1MeTIQ did not change the dopamine level in basal samples; however, a weak increase in dopamine release after the last dose of 1MeTIQ (up to $200 \% ; \mathrm{F}[1,16]=1.63$; N.S.) was observed (Fig. 4b). Interestingly, in the combined treatment group, chronic injection of 1MeTIQ completely blocked the effect induced by repeated administrations of 1BnTIQ (Fig. 4b). The concentration of dopamine in basal samples was slightly above the control (saline) group, and after the final injection of 1MeTIQ and 1BnTIQ, the dopamine release increased by approximately $200 \%$ (Fig. 4b).

\section{The Effect of Chronic 1BnTIQ Administration on 3 -MT release in vivo}

Duncan's test indicated that chronic 1BnTIQ administration did not change the level of 3-MT (Figs. 3a, b, 5a, b).

\section{The Effect of a Single or Chronic Administration of 1 MeTIQ on 1BnTIQ-Induced Changes on the Concentration of 3-MT in the Rat Striatum}

As shown in Fig. 5a, acute 1MeTIQ (50 mg/kg i.p.) administration produced a significant $(\mathrm{F}[1,17]=30.86$; $p<0.05$ ) increase in the concentration of 3-MT in the rat striatum (up to $500 \%$ ). This effect was strongly potentiated in the joint treatment group when 1MeTIQ was given with the last dose of 1BnTIQ (the concentration of 3-MT was elevated approximately $2000 \% ; \mathrm{F}[1,17]=14.03$; $p<0.01$ ) (Fig. 5a).

In contrast to the effect of chronic treatment with 1BnTIQ, multiple $1 \mathrm{MeTIQ}(50 \mathrm{mg} / \mathrm{kg}$ i.p.) administrations produced a huge and significant $(\mathrm{F}[1,17]=52.41$; $p<0.01)$ increase in the concentration of 3-MT in the rat striatum (up to $2000 \%$ ) (Fig. 5b). A similar effect was observed in the mixed group (with chronic treatment 
Fig. 3 The effect of acute (a) or chronic (b) administration of TIQ on 1BnTIQ-induced changes in 3-MT concentration in the rat striatum, 1BnTIQ was administered chronically (1BnTIQch) at a dose of $25 \mathrm{mg} /$ $\mathrm{kg}$ i.p. for 14 consecutive days. In the mixed group, TIQ $(50 \mathrm{mg} / \mathrm{kg}$ i.p.) was given acutely (TIQa) a $20 \mathrm{~min}$ before the last 1BnTIQ administration or chronically (TIQch), b 20 min before each 1BnTIQ administration. The control group was treated with saline. The dialysate was collected every $20 \mathrm{~min}$. The concentration of extraneuronal metabolite of dopamine 3-MT was measured. The data are expressed as the mean \pm SEM $(n=5-6)$. Statistical significance: $* p<0.05$, $* * p<0.01$ from the basal value; ${ }^{+} p<0.05$ versus 1BnTIQ-treated group (Duncan's test)

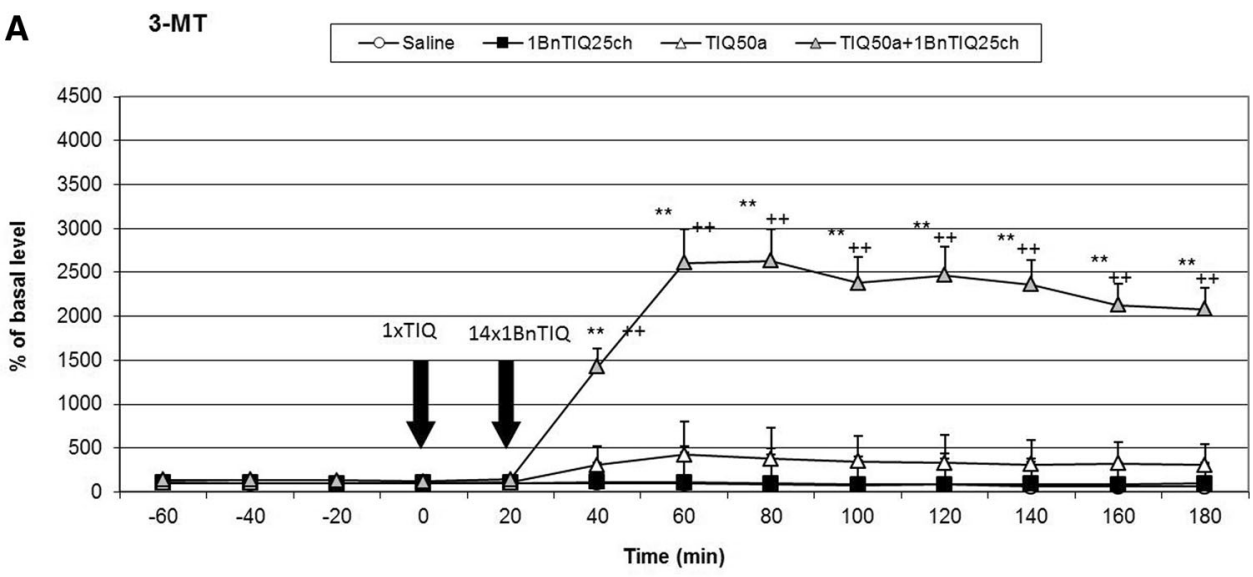

B 3-MT $\rightarrow 0-$ Saline $\rightarrow-1 \mathrm{BnTIQ} 25 \mathrm{ch} \rightarrow-\mathrm{TIQ50ch} \triangle-\mathrm{TIQ} 50 \mathrm{ch}+1 \mathrm{BnTIQ} 25 \mathrm{ch}$

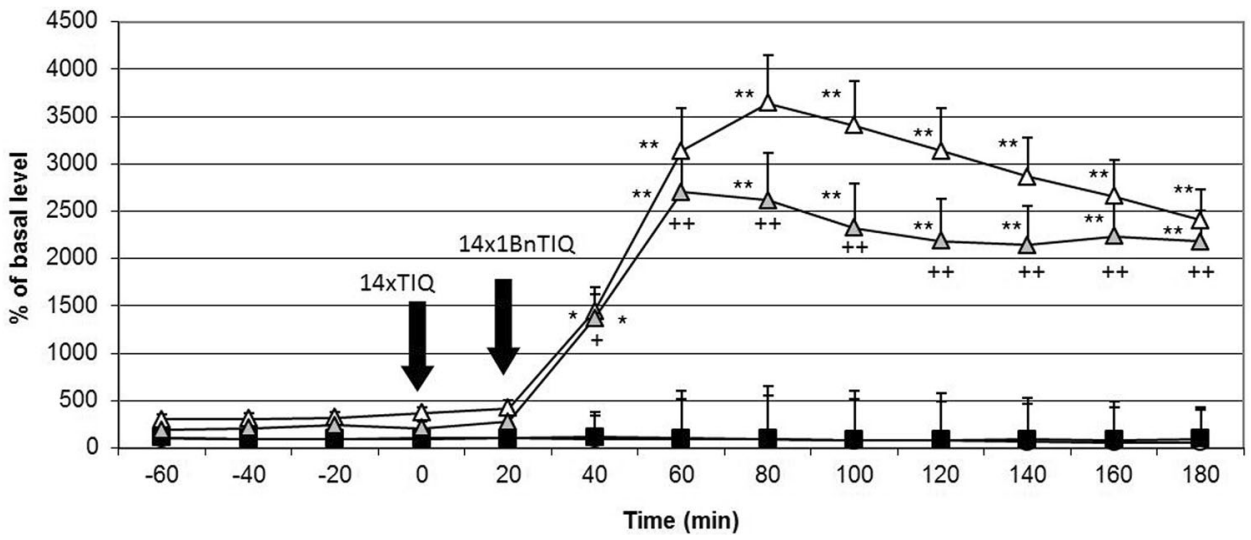

1MeTIQ and 1BnTIQ), and the concentration of 3-MT was increased up to $2000 \%(\mathrm{~F}[1,17]=47.15 ; p<0.01)$ (Fig. 5b).

\section{Discussion}

The chronic administration of 1BnTIQ produced clear and significant disturbances in the function of dopaminergic neurons, which led to an increase in dopamine release in the striatum. Based on our previous experiments, we confirmed that chronic treatment with a low dose of 1 BnTIQ $(25 \mathrm{mg} / \mathrm{kg}$ i.p.) damaged the dopamine storage mechanisms causing its non-physiological rise in the extracellular space in the rat striatum (Wassik et al. 2009, 2014). The main finding of the present study is that chronic administration of TIQ and 1MeTIQ completely prevented the disorders on dopamine release induced by multiple administrations of 1BnTIQ. Dopamine, the main neurotransmitter involved in motor control and Parkinson's disease, is metabolized both intra- and extraneuronally. The extraneuronal dopamine metabolite, 3-MT, which is present in the synaptic cleft at relatively low concentrations comparable to dopamine, is considered to be a marker of dopamine release (Karoum et al. 1994). However, in contrast to intraneuronal dopamine metabolite, DOPAC and the final metabolite HVA, 3-MT may be biologically active. It was demonstrated previously that 3-MT has a considerable affinity as antagonist for noradrenergic $\alpha 1$ and dopamine D1 and D2 receptors in rat brain (AntkiewiczMichaluk et al. 2008). In the behavioral tests chronic administration of 1BnTIQ-induced hyperactivity, while multiple injection of both, TIQ and 1MeTIQ significantly decreased the rats locomotor activity (Fig. 1a, b). Additionally, in the combined groups, TIQ and 1MeTIQ inhibited 1-BnTIQ-induced hyperactivity. In that light, the mechanism of the action of TIQ and 1MeTIQ, leading to an increase the concentration of 3-MT antagonism to 1BnTIQ-produced hyperactivity may be explained by its inhibition to catecholaminergic receptors.

Although all substances used in the present study belong to the same chemical group, their mechanisms of action are completely opposite. 1BnTIQ activates the dopamine oxidation pathway, which leads to the elevation in free radical 
Fig. 4 The effect of acute (a) or chronic (b) administration of 1MeTIQ on 1BnTIQ-induced changes in dopamine release in the rat striatum, 1BnTIQ was administered chronically (1BnTIQch) at a dose of $25 \mathrm{mg}$ / $\mathrm{kg}$ i.p. for 14 consecutive days. In the mixed group, 1MeTIQ (50 $\mathrm{mg} / \mathrm{kg}$ i.p.) was given acutely (1MeTIQa) a $20 \mathrm{~min}$ before the last 1BnTIQ administration or chronically (1MeTIQch), b 20 min before each 1BnTIQ administration. The control group was treated with saline. The dialysate was collected every $20 \mathrm{~min}$. The concentration of dopamine (DA) was measured. The data are expressed as the mean \pm SEM $(n=5-6)$. Statistical significance: $* p<0.05, * * p<0.01$ from the basal value; ${ }^{+} p<0.05$ versus 1BnTIQ-treated group (Duncan's test)
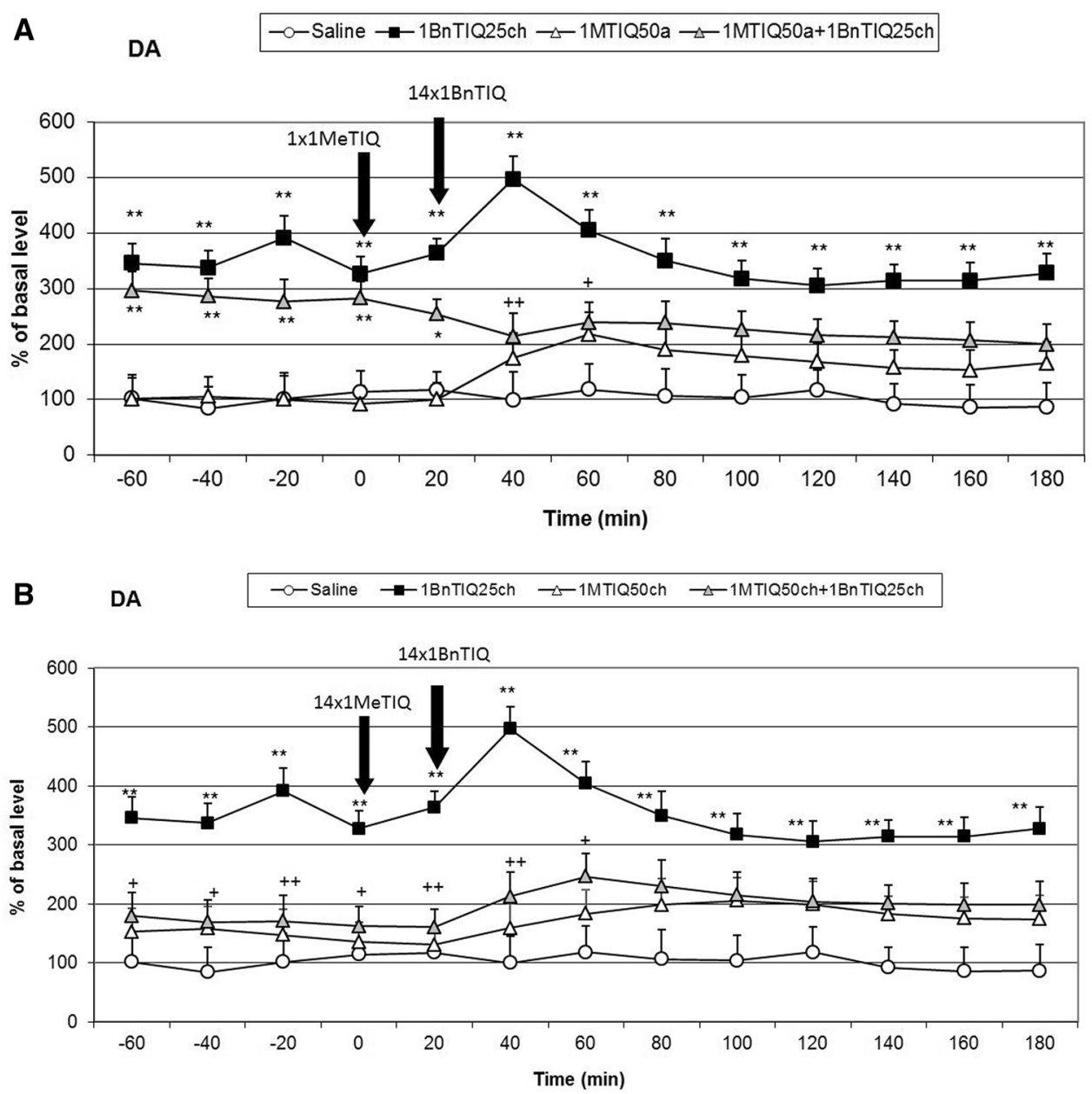

production (Waasik et al. 2014). On the other hand, TIQ and 1MeTIQ exhibit a contrasting molecular mechanism, and act as reversible MAO inhibitors, blocking the production of free radicals and activating the COMT-dependent O-methylation pathway (Antkiewicz-Michaluk et al. 2001; Wąsik et al. 2009). Our present in vivo study demonstrated that multiple administrations of 1BnTIQ produced a significant and long-lasting increase (approximately $300 \%$ in comparison to the saline group) in dopamine release in the rat striatum, measured in the basal samples $24 \mathrm{~h}$ after its 13th injection ( $1 \mathrm{~h}$ before the last 14th injection). Such permanent increase in dopamine levels in the extracellular space after the chronic administrations of 1BnTIQ may, like the treatment with psychostimulants, leads to neurotoxic effects. After the 14th (last) dose of 1BnTIQ, dopamine release was elevated up to $500 \%$ of the saline group; however, at the same time, no change in 3-MT concentration was observed. Moreover, 3-MT is considered as the most reliable indicator of dopamine release into the synaptic cleft (Egan et al. 1991; Karoum et al. 1994). Additionally, as demonstrated previously, 3-MT, in contrast to DOPAC and HVA, is an active metabolite of dopamine and possesses its own receptor activity (Antkiewicz-Michaluk et al. 2008; Alachkar et al. 2010). It is well known that elevation of dopamine release induces an increase of dopaminergic activity and leads to hyperactivity. The results of the in vivo microdialysis study are in agreement with the behavioral locomotor activity test in which chronic treatment with 1 BnTIQ-produced hyperactivity in rats (Fig. 1a, b).

As we demonstrated earlier, 1BnTIQ similarly to reserpine (a specific vesicular monoamine transporter 2 (VMAT2) inhibitor) significantly depleted striatal dopamine (Wasik et al. 2009). We postulated that 1BnTIQ might damage VMAT2 in dopaminergic neurons, leading to the pathological release of dopamine into the cytosol, and increased its MAO-dependent oxidation and free radical production (Wasik et al. 2014). On the other hand, 1BnTIQ in low micromolar concentrations significantly inhibited the dopamine reuptake in slices of the rat striatum (Patsenka et al. 2004). Similarly, Okada et al. (1998) demonstrated that 1BnTIQ inhibited the uptake of $[3 \mathrm{H}]-$ dopamine by the dopamine transporter expressed in HEK293 cells. We suggest that DAT is responsible for the 
Fig. 5 The effect of acute (a) or chronic (b) administration of 1MeTIQ on 1BnTIQ-induced changes in 3-MT concentration in the rat striatum, 1BnTIQ was administered chronically (1BnTIQch) at a dose $25 \mathrm{mg} / \mathrm{kg}$ i.p. for 14 consecutive days. In the mixed group, 1MeTIQ (50 $\mathrm{mg} / \mathrm{kg}$ i.p.) was given acutely (1MeTIQa) a $20 \mathrm{~min}$ before last 1BnTIQ administration or chronically (1MeTIQch), b 20 min before each 1BnTIQ administration. The control group was treated with saline. The dialysate was collected every $20 \mathrm{~min}$. The concentration of extraneuronal metabolite of dopamine 3-MT was measured. The data are expressed as the mean \pm SEM $(n=5-6)$. Statistical significance: $* p<0.05$, $* * p<0.01$ from the basal value; ${ }^{+} p<0.05$ versus 1BnTIQ-treated group (Duncan's test)
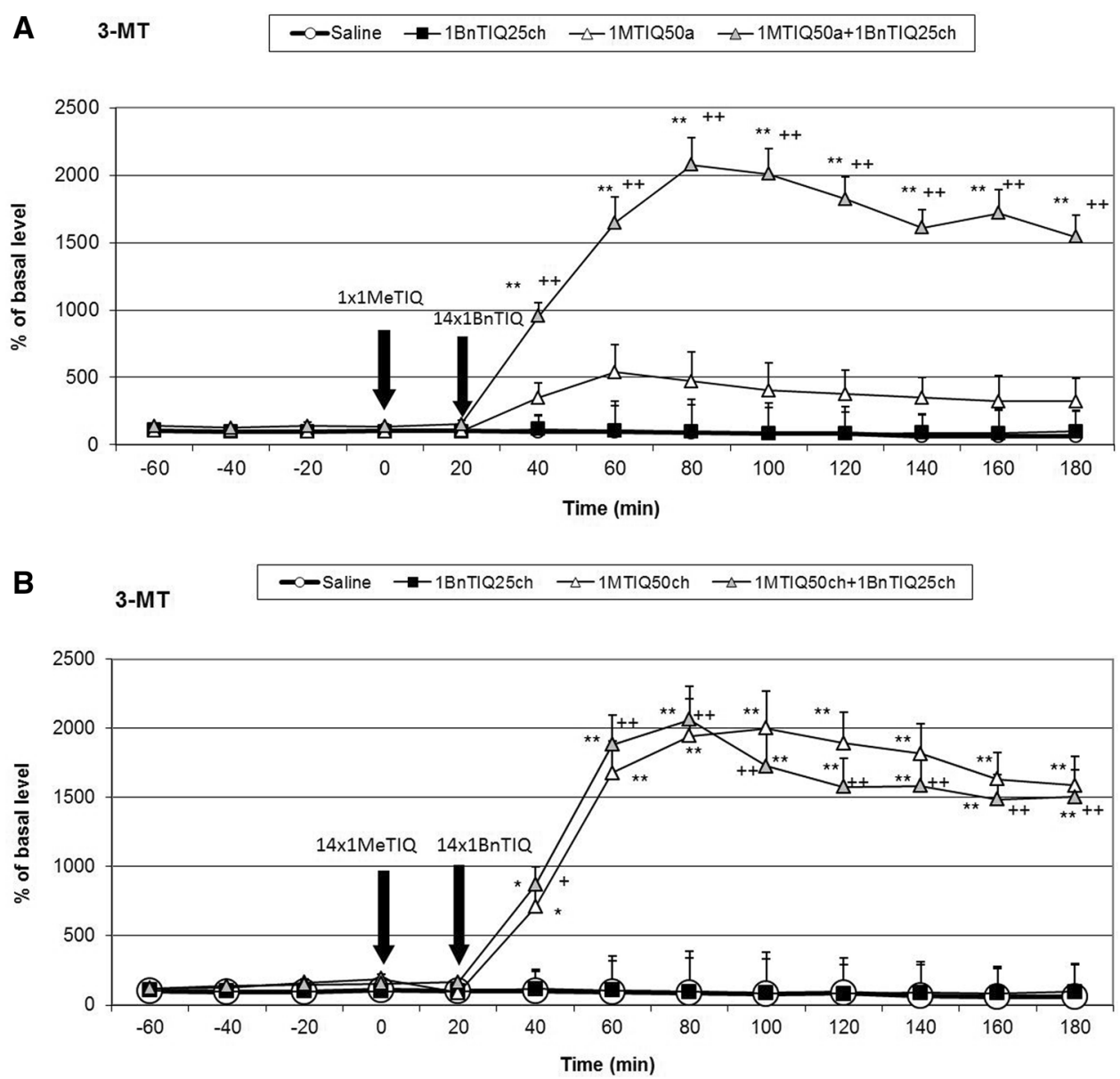

selective transport of 1BnTIQ into the dopaminergic neurons, leading to the 1BnTIQ neurotoxicity correlated with impaired dopamine storage via inhibition of VMAT2 (Wasik et al. 2009). Since TIQ and 1MeTIQ have also affinity to DAT, both substances may restrict access of 1BnTIQ to the DAT (Patsenka et al. 2004). This mechanism results in a reduction of penetration of 1 BnTIQ into the neurons. The present study revealed that acute treatment with TIQ induced an increase in dopamine release (approximately $300 \%$ ), while 1MeTIQ showed a similar effect of a lesser magnitude (Figs. 2a, 4a). Taking into account that both investigated compounds, TIQ and $1 \mathrm{MeTIQ}$, act as reversible MAO inhibitors, they shift dopamine catabolism toward the COMT-dependent O-methylation pathway, which leads to a significant increase in the extraneuronal concentration of the dopamine metabolite 3-MT (Antkiewicz-Michaluk et al. 2001; Patsenka and Antkiewicz-Michaluk 2004). In the present paper, such properties of TIQ and 1MeTIQ were confirmed in our in vivo microdialysis studies which demonstrated an increase in 3-MT level by approximately $500 \%$ in the case of acute administration and up to $2500 \%$ versus saline group after chronic treatment. It is worth emphasizing, as previously demonstrated that 3-MT, an extraneuronal dopamine metabolite, shows affinity for $\alpha 1$-adrenergic and D1 and D2 receptors as an antagonist, and may play an important role as an inhibitory regulator, counteracting the excessive stimulation of catecholaminergic neurons (Antkiewicz-Michaluk et al. 2008; Alachkar et al. 2010). Thus, a high concentration of 3-MT may play an important role in locomotor activity as dopamine D2 and noradrenergic $\alpha 1$ receptors antagonists (Antkiewicz-Michaluk et al. 2008). Such mechanism of action of 3-MT leads to the paradoxical effect, where despite the increase in dopamine release, we observed decrease in the locomotor activity in rats after treatment with both, TIQ and 1MeTIQ (Fig. 1a, b).

In the combined treatment group, an acute dose of TIQ given before the last dose of 1BnTIQ only slightly reduced the effect of 1BnTIQ, whereas acute administration of 1MeTIQ produced a clear decrease in dopamine release (Figs. 2a, 4a). In both combined treatment groups, a massive increase in 3-MT levels was observed (2500 and $2000 \%$, respectively; Figs. 3a, 5a). Such an increase in the 3-MT concentration may be associated with the retention of dopamine in the extracellular space, due to dopamine reuptake blockade by TIQ and 1MeTIQ, and catabolism of 
all available dopamine by the COMT-dependent O-methylation pathway. It is worth emphasizing that such a mechanism of action for TIQ and 1MeTIQ inhibits the production of free radicals, which are formed during the catabolism of dopamine by MAO-dependent oxidation, and consequently leads to a reduction in oxidative stress (Antkiewicz-Michaluk et al. 2006; Miller et al. 1996; Patsenka and Antkiewicz-Michaluk 2004).

Interestingly, in both combined treatment groups, chronic treatment with TIQ or 1MeTIQ completely antagonized the 1BnTIQ-induced pathological increase in dopamine release in the basal samples (Figs. 2b, 4b). Simultaneously, in these groups, we observed a huge elevation in the concentration of 3-MT after the last dose of TIQ and 1MeTIQ (up to 2500 and $2000 \%$, respectively) (Figs. 3b, 5b). These results are important because they demonstrate that chronic administration of both TIQ and 1MeTIQ can counteract the disturbances in dopamine release produced by multiple administrations of 1BnTIQ. We suggest that TIQ and 1MeTIQ given before each 1BnTIQ injection inhibit DAT and in this way block penetration of $1 B n T I Q$ into the neurons. Since $1 B n T I Q$ penetrates into cells via DAT, DAT is inhibited by prior injection of TIQ and 1MeTIQ; therefore, in the combined treatment groups, we did not observe the effect of 1BnTIQ (Patsenka et al. 2004). The data from the behavioral tests (locomotor activity) confirmed that a high concentration of 3-MT, which was observed in both joint treatment groups, acted as an endogenous neuroleptic, as previously demonstrated (Antkiewicz-Michaluk et al. 2008), and could block hyperactivity in rats (Fig. 1a, b). It is important to mention that pronounced increases in dopamine O-methylation in the COMT-dependent pathway may provide neuroprotection (Antkiewicz-Michaluk et al. 2001; Miller et al. 1996). It was also indicated that elevation of 3-MT in the process of dopamine O-methylation protected cells against oxidative stress (Miller et al. 1996). The neuroprotective properties of $1 \mathrm{MeTIQ}$ against different dopaminergic neurotoxins, including MPTP, 6-OHDA, rotenone, and 1BnTIQ, were also demonstrated in in vitro studies in cultured mesencephalic neurons (Kotake et al. 2005) and in ex vivo studies (Antkiewicz-Michaluk et al. 2011; Wąsik et al. 2016).

\section{Conclusions}

Summing up the results, based on the in vivo microdialysis study presented in this paper, we suggest that both endogenous amines TIQ and 1MeTIQ can antagonize 1BnTIQ-induced clear disorders of dopamine release in vivo in rat striatum. Furthermore, these amines were able to restore the physiological functions of striatal dopamine neurons disturbed by chronic application of $1 \mathrm{BnTIQ}$.

Acknowledgments The authors would like to thank Maria Kafel and Krzysztof Michalski for their technical assistance.

Funding This study was financially supported through a grant from the National Science Centre Grant No. DEC-2012/07/B/NZ7/01149, and statutory funds from the Institute of Pharmacology, Polish Academy of Sciences, Krakow, Poland.

\section{Compliance with Ethical Standards}

Conflict of Interest The authors declare that the research was conducted in the absence of any commercial or financial relationships that could be construed as a potential conflict of interest.

Open Access This article is distributed under the terms of the Creative Commons Attribution 4.0 International License (http://crea tivecommons.org/licenses/by/4.0/), which permits unrestricted use, distribution, and reproduction in any medium, provided you give appropriate credit to the original author(s) and the source, provide a link to the Creative Commons license, and indicate if changes were made.

\section{References}

Abe K, Taguchi K, Wasai T, Ren J, Utsunomiya I, Shinohara T, Miyatake T, Sano T (2001a) Biochemical and pathological study of endogenous 1-benzyl-1,2,3,4-tetrahydroisoquinoline-induced parkinsonism in the mouse. Brain Res 907:134-138

Abe K, Taguchi K, Wasai T, Ren J, Utsunomiya I, Shinohara T, Miyatake T, Sano T (2001b) Stereoselective effect of (R)- and (S)- 1-methyl-1,2,3,4-tetrahydroisoquinolines on a mouse model of Parkinson's disease. Brain Res Bull 56:55-60

Abe K, Saitoh T, Horiguchi Y, Utsunomiya I, Taguchi K (2005) Synthesis and neurotoxicity of tetrahydroisoquinoline derivatives for studying Parkinson's disease. Biol Pharm Bull 28:1355-1362

Alachkar A, Brotchie JM, Jones OT (2010) Binding of dopamine and 3-methoxytyramine as L-DOPA metabolites to human $\alpha 2$ adrenergic and dopaminergic receptors. Neurosci Res 67: 245-249

Antkiewicz-Michaluk L, Michaluk J, Mokrosz M, Romańska I, Lorenc-Koci E, Otha S, Vetulani J (2001) Different action on dopamine catabolic pathways of two endogenous 1,2,3,4tetrahydroisoquinolines with similar antidopaminergic properties. J Neurochem 78:100-108

Antkiewicz-Michaluk L, Karolewicz B, Romańska I, Michaluk J, Bojarski AJ, Vetulani J (2003) 1-Methyl-1,2,3,4-tetrahydroisoquinoline protects against rotenone-induced mortality and biochemical changes in rat brain. Eur J Pharmacol 466:263-269

Antkiewicz-Michaluk L, Wardas J, Michaluk J, Romańska I, Bojarski A, Vetulani J (2004) Protective effect of 1-methyl-1,2,3,4tetrahydroisoquinoline against dopaminergic neurodegeneration in the extrapyramidal structures produced by intracerebral injection of rotenone. Inter J Neuropsychopharmacol 7:155-163

Antkiewicz-Michaluk L, Łazarewicz JW, Patsenka A, Kajta M, Ziemińska E, Salińska E, Wąsik A, Gołembiowska K, Vetulani J (2006) The mechanism of 1,2,3,4-tetrahydroisoquinolines neuroprotection: the importance of free radicals scavenging properties and inhibition of glutamate-induced excitotoxicity. J Neurochem 97:846-856 
Antkiewicz-Michaluk L, Ossowska K, Romańska I, Michaluk J, Vetulani J (2008) 3-Methoxytyramine, an extraneuronal dopamine metabolite plays a physiological role in the brain as an inhibitory regulator of catecholaminergic activity. Eur J Pharmacol 599:32-35

Antkiewicz-Michaluk L, Wąsik A, Romańska I, Michaluk J (2011) Both stereoselective (R)- and (S)-1-methyl-1,2,3,4-tetrahydroisoquinoline enantiomers protects striatal terminals against rotenone-induced suppression of dopamine release. Neurotox Res 20:134-149

Egan MF, Karoum F, Wyatt RJ (1991) Effects of acute and chronic clozapine and haloperidol administration on 3-methoxytyramine accumulation in the rat prefrontal cortex, nucleus accumbens and striatum. Eur J Pharmacol 199:191-199

Karoum F, Chrapusta SJ, Egan MF (1994) 3-Methoxytyramine is the major metabolite of released dopamine in the rat frontal cortex: reassessment of the effects of antipsychotics on the dynamics of dopamine release and metabolism in frontal cortex, nucleus accumbens and striatum by a simple two pool model. J Neurochem 63:972-978

Kohta R, Kotake Y, Hosoya T, Hiramatsu T, Otsubo Y, Koyama H, Hirokane Y, Yokoyama Y, Ikeshoji H, Oofusa K, Suzuki M, Ohta S (2010) 1-Benzyl-1,2,3,4-tetrahydroisoquinoline binds with tubulin beta, a substrate of parkin, and reduces its polyubiquitination. $\mathrm{J}$ Neurochem 114:1291-1301

Kotake Y, Tasaki Y, Makino Y, Otha S, Hirobe M (1995) 1-Benzyl1,2,3,4-tetrahydroisoquinoline as a parkinsonism-inducing agent: a novel endogenous amine in mouse brain and parkinsonian CSF. J Neurochem 65(6):2633-2638

Kotake Y, Ohta S, Kanazawa I, Sakurai M (2003) Neurotoxicity of an endogenous brain amine, 1-benzyl-1,2,3,4-tetrahydroisoquinoline, in organotypic slice co-culture of mesencephalon and striatum. Neuroscience 117:63-70

Kotake Y, Taguchi R, Okuda K, Sekiya Y, Tasaki Y, Hirobe M, Ohta S (2005) Neuroprotective effect of 1-methyl-1,2,3,4-tetrahydroisoquinoline on cultured rat mesencephalic neurons in the presence or absence of various neurotoxins. Brain Res 1033(2):143-150

Miller J, Selhub J, Joseph J (1996) Oxidative damage caused by free radicals produced during catecholamine autooxidation: protective effects of O-methylation and melatonin. Free Radic Biol Med 21:241-249

Okada T, Shimada S, Sato K, Kotake Y, Kawai H, Ohta S, Tohyama M, Nishimura T (1998) Tetrahydropapaveroline and its derivatives inhibit dopamine uptake through dopamine transporter expressed in HEK293 cells. Neurosci Res 30:87-90

Patsenka A, Antkiewicz-Michaluk L (2004) Inhibition of rodent brain monoamine oxidase and tyrosine hydroxylase by endogenous compounds-1,2,3,4-tetrahydro-isoquinoline alkaloids. Pol J Pharmacol 56:727-734

Patsenka A, Michaluk J, Antkiewicz-Michaluk L (2004) 1,2,3,4Tetrahydroisoquinoline alkaloids as endogenous inhibitors of brain monoamine oxidase, tyrosine hydroxylase and uptake of monoamines: in vitro study. In 13th international symposium on molecular and physiological aspects of regulatory processes of the organism. Kraków, Poland, Materials 2004, p 344

Shavali S, Ebadi M (2003) 1-Benzyl-1,2,3,4-tetrahydroisoquinoline (1BnTIQ), an endogenous neurotoxin, induces dopaminergic cell death through apoptosis. Neurotoxicology 24:417-424

Singer TP, Ramsay RR (1995) Flauoprotein structure and mechanism 2. Monoamine oxidases: old friends hold many surprises. FASEB J 9(8):605-610

Wąsik A, Romańska I, Antkiewicz-Michaluk L (2009) 1-Benzyl1,2,3,4-tetrahydroisoquinoline, an endogenous parkinsonism-inducing toxin, strongly potentiates MAO-dependent dopamine oxidation and impairs dopamine release: ex vivo and in vivo neurochemical studies. Neurotox Res 15:23-31

Wąsik A, Romańska I, Michaluk J, Kajta M, Antkiewicz-Michaluk L (2014) 1-Benzyl-1,2,3,4-tetrahydroisoquinoline, an endogenous neurotoxic compound, disturbs the behavioral and biochemical effects of 1-DOPA: in vivo and ex vivo studies in the rat. Neurotox Res 26(3):240-254

Wąsik A, Romańska I, Michaluk J, Zelek-Molik A, Nalepa I, Antkiewicz-Michaluk L (2016) Neuroprotective effect of endogenous amine 1MeTIQ in animals model of Parkinson's disease. Neurotox Res 29:351-363

Yamakawa T, Ohta S (1997) Isolation of 1-methyl-1,2,3,4-tetrahydroisoquinoline-synthesizing enzyme from rat brain: a possible Parkinson's disease-preventing enzyme. Biochem Biophys Res Commun 236:676-681

Yamakawa T, Ohta S (1999) Biosynthesis of a parkinsonismpreventing substance, 1-methyl-1,2,3,4-tetrahydroisoquinoline, is inhibited by parkinsonism-inducing compounds in rat brain mitochondrial fraction. Neurosci Lett 259:157-160

Yamakawa T, Kotake Y, Fujitani M, Shintani H, Makino Y, Ohta S (1999) Regional distribution of parkinsonism-preventing endogenous tetrahydroisoquinoline derivatives and an endogenous parkinsonism-preventing substance-synthesizing enzyme in monkey brain. Neurosci Lett 276:68-70 